\title{
Learning in 3D with Pymol and Proteopedia during a pandemic: enzyme catalysis and conformational change
}

\author{
Roderico Acevedo and Karsten Theis \\ Chemical and Physical Sciences, Westfield State University \\ ktheis@westfield.ma.edu
}

Crystal structures of proteins are three-dimensional, but most depictions of them, in textbooks and in the scientific literature, are not. When students are on campus, they can interact with physical models, discuss structures in the computer lab and experience the properties and functions of proteins in the biochemistry lab. We describe two projects that support interactive, collaborative and experiential learning in a remote setting. In the first project, students explored metabolic enzymes using the visualization software Pymol. Starting with crystal structures in the Protein Data Bank, students learned the basics of Pymol: they superimposed structures representing different stages in the catalytic mechanism, highlighted non-covalent interactions, identified bonds broken and made, and discussed the active sites of these enzymes in the context of the protein fold. In weekly meetings, students shared their progress and setbacks amongst each other, and used peer-to-peer learning to elevate their chemical and graphical design skills. Individually, they created different scenes and made them into a short video for which they provided an explanatory voiceover. Students wrote about their progress in weekly reflections. Many students reported being "excited and challenged" about learning a new technique at the outset. Later, deeper learning strategies emerged such as searching the primary literature or comparing existing videos to see how one might position an active site. The help-seeking behavior also became more sophisticated, for example asking for a video tutorial showing how to add or remove functional groups from a model. Overall, students were actively engaged in their projects and were eager to share what they had learned in discussions with their peers. The second project, housed on the public science site Proteopedia.org, aims at presenting examples of conformational change in a more interactive way. We wrote a series of Jmol scripts (storymorph.spt) to make it easier to superimpose structures and create morphs (fictional trajectories connecting conformational states). Using an algorithm that combines rigid-body movement with linear interpolation, morphs are made on the fly, allowing the visitor to change parameters (such as the timing of distinct parts of the conformational change or the initial superposition) to get a better feel for how the conformation might change. It is also possible to slow down or pause the morph, allowing visitors to explore the suggested intermediates in three-dimensions, including potential clashes or unrealistic bond lengths or angles. Morphs made available through this project include hexokinase binding to glucose, RNA polymerase transitioning from early to late initiation, conformational changes in calmodulin, and the pre-fusion to post-fusion transition of the coronavirus spike protein. Together these two projects highlight simple ways to keep science-learning interactive, collaborative, fun, and - most importantly - threedimensional in spite of the limitations caused by a pandemic.

Keywords: catalysis, conformational change, interactive learning 